\title{
Frontiers in endovascular thrombectomy for ischemic stroke
}

\author{
Matthew Linger ${ }^{1}$, Bruce C. V. Campbell1,2 \\ 'Department of Medicine and Neurology, Melbourne Brain Centre at the Royal Melbourne Hospital, University of Melbourne, \\ Parkville, Victoria 3050, Australia. \\ ${ }^{2}$ The Florey Institute of Neuroscience and Mental Health, University of Melbourne, Parkville, Victoria 3010, Australia.
}

Correspondence to: Prof. Bruce C. V. Campbell, Department of Neurology, Royal Melbourne Hospital, 300 Grattan Street, Parkville, Victoria 3050, Australia. E-mail: bruce.campbell@mh.org.au

How to cite this article: Linger M, Campbell BCV. Frontiers in endovascular thrombectomy for ischemic stroke. Vessel Plus 2020;4:36. http://dx.doi.org/10.20517/2574-1209.2020.51

Received: 15 Sep 2020 First Decision: 9 Oct 2020 Revised: 25 Oct 2020 Accepted: 5 Nov 2020 Published: 18 Nov 2020

Academic Editor: Aaron S. Dumont Copy Editor: Cai-Hong Wang Production Editor: Jing Yu

\begin{abstract}
Stroke is a leading cause of morbidity and mortality worldwide. There have been significant advances in the hyperacute treatment of patients with ischemic stroke with the advent and application of reperfusion therapies, including intravenous thrombolysis and endovascular thrombectomy. Endovascular thrombectomy involves the removal of thrombus from an artery using a mechanical retriever or aspiration with angiographic visualization. This review aims to outline the current evidence to support the use of endovascular thrombectomy and highlight areas of ongoing research.
\end{abstract}

Keywords: Ischemic stroke, endovascular thrombectomy, thrombolysis, tenecteplase, tissue plasminogen activator, alteplase, computed tomography perfusion, magnetic resonance imaging, randomized trial

\section{EVIDENCE FOR ENDOVASCULAR THROMBECTOMY AND THE TIME WINDOW FOR TREATMENT}

The benefit of endovascular thrombectomy over standard medical care was proven in 2015, with 5 trials [Multicenter Randomized Clinical Trial of Endovascular Treatment for Acute Ischemic Stroke in the Netherlands (MR CLEAN); Extending the Time for Treatment in Emergency Neurological Deficits Intra-Arterial (EXTEND-IA); Endovascular Treatment for Small Core and Anterior Circulation Proximal

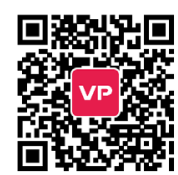


Occlusion with Emphasis on Minimizing CT to Recanalization Times (ESCAPE); Solitaire With the Intention For Thrombectomy PRIMary Endovascular Treatment (SWIFT PRIME); and Randomized Trial of Revascularization with Solitaire FR Device vs. Best Medical Therapy in the Treatment of Acute Stroke Due to Anterior Circulation Large Vessel Occlusion Presenting within Eight Hours of Symptom Onset (REVASCAT)] demonstrating benefit in patients presenting with large vessel occlusion (LVO) of the anterior circulation within $6 \mathrm{~h}$ of symptom onset ${ }^{[1-5]}$. LVO in the anterior circulation is defined as an occlusion of the intracranial internal carotid artery and/or the first segment of the middle cerebral artery (M1). Patients with intracranial occlusion and tandem extracranial carotid artery occlusion were also included in the trials and clearly benefitted. In an individual patient data meta-analysis of these trials, the rate of functional independence [defined as a modified Rankin scale (mRS) score of 0-2] was significantly higher in the thrombectomy cohort (46\%) compared to medical therapy (27\%), equaling a numberneeded-to-treat (NNT) of $5^{[6]}$. There was a similar rate of serious adverse events, in particular symptomatic intracranial hemorrhage, between thrombectomy and medical therapy groups ${ }^{[6]}$. These results were in contrast to 3 previous trials published in 2013 that failed to show clinical benefit of thrombectomy ${ }^{[7-9]}$. The difference in outcomes is likely explained by improvements in patient selection (detecting LVO with noninvasive imaging prior to angiography), faster door-to-arterial access times and better devices to achieve faster and more complete reperfusion ${ }^{[10]}$. The THRACE (Mechanical Thrombectomy After Intravenous Alteplase vs. Alteplase Alone After Stroke), PISTE (Pragmatic Ischaemic Stroke Thrombectomy Evaluation) and RESILIENT (Randomisation of endovascular treatment with stent-retriever and/or thromboaspiration vs. best medical therapy with acute ischemic stroke due to large vessel occlusion) trials were also subsequently reported with consistent results in favor of thrombectomy ${ }^{[11-13]}$.

Data from the MR CLEAN trial indicate that the benefit of thrombectomy, when patient selection is simply based on evidence of LVO using non-invasive imaging, is strongly time-dependent and ceases to be statistically significant $\sim 6 \mathrm{~h}$ after onset ${ }^{[14]}$. The HERMES (Highly Effective Reperfusion Evaluated in Multiple Endovascular Stroke Trials) pooled data found statistically significant benefit out to $7.3 \mathrm{~h}$ but included patients selected on the basis of good collateral flow or favorable CT perfusion which has subsequently been shown to extend the time window for treatment ${ }^{[15]}$.

The DAWN trial (DWI or CTP Assessment with Clinical Mismatch in the Triage of Wake-Up and Late Presenting Strokes Undergoing Neurointervention with Trevo) in patients 6-24 h after the time they were last known to be well used clinical-core mismatch imaging selection. This involved CT perfusion or diffusion MRI to identify a relatively small volume of irreversibly injured brain (ischemic core) compared to the severity of clinical deficit and also factored in patient age ${ }^{[16]}$. CT perfusion has been shown to achieve similar perfusion mismatch classification compared to MRI ${ }^{[17]}$. Volumetric agreement studies have shown that overestimation of ischemic core using a relative cerebral blood flow threshold $<30 \%$ of normal brain ${ }^{[18]}$ is uncommon and tends to involve white matter in patients with ultra-rapid reperfusion ${ }^{[19-21]}$. The DEFUSE 3 trial (Endovascular Therapy Following Imaging Evaluation for Ischemic Stroke) in patients 6-16 h after the time they were last known to be well used CT perfusion or MRI perfusion-diffusion mismatch and required $<70 \mathrm{~mL}$ irreversibly injured ischemic core combined with the critically hypoperfused region being both $>15 \mathrm{~mL}$ and $>1.8$ times the volume of the ischemic core ${ }^{[22]}$. In the DAWN and DEFUSE 3 trials, the NNT for functional independence (defined as mRS score 0-2) was 2.8 and 3.6, respectively ${ }^{[16,22]}$. There was no significant difference in the rate of symptomatic intracerebral hemorrhage between thrombectomy and medical therapy groups in these trials. The DEFUSE 3 inclusion criteria classified $\sim 60 \%$ more patients as eligible for thrombectomy than the DAWN criteria and these additional patients benefitted at least as much from the thrombectomy procedure ${ }^{[22]}$. Perfusion imaging selection is therefore recommended in international guidelines for selection of patients for thrombectomy 6-24 $\mathrm{h}$ after the time they were last known to be well [Figure 1] ${ }^{[23,24]}$. Relatively few patients present with LVO beyond $24 \mathrm{~h}$, and a trial is unlikely to be feasible. However, anecdotal reports have suggested potential benefit in patients $>24 \mathrm{~h}$ after onset if imaging is favorable ${ }^{[25]}$. 


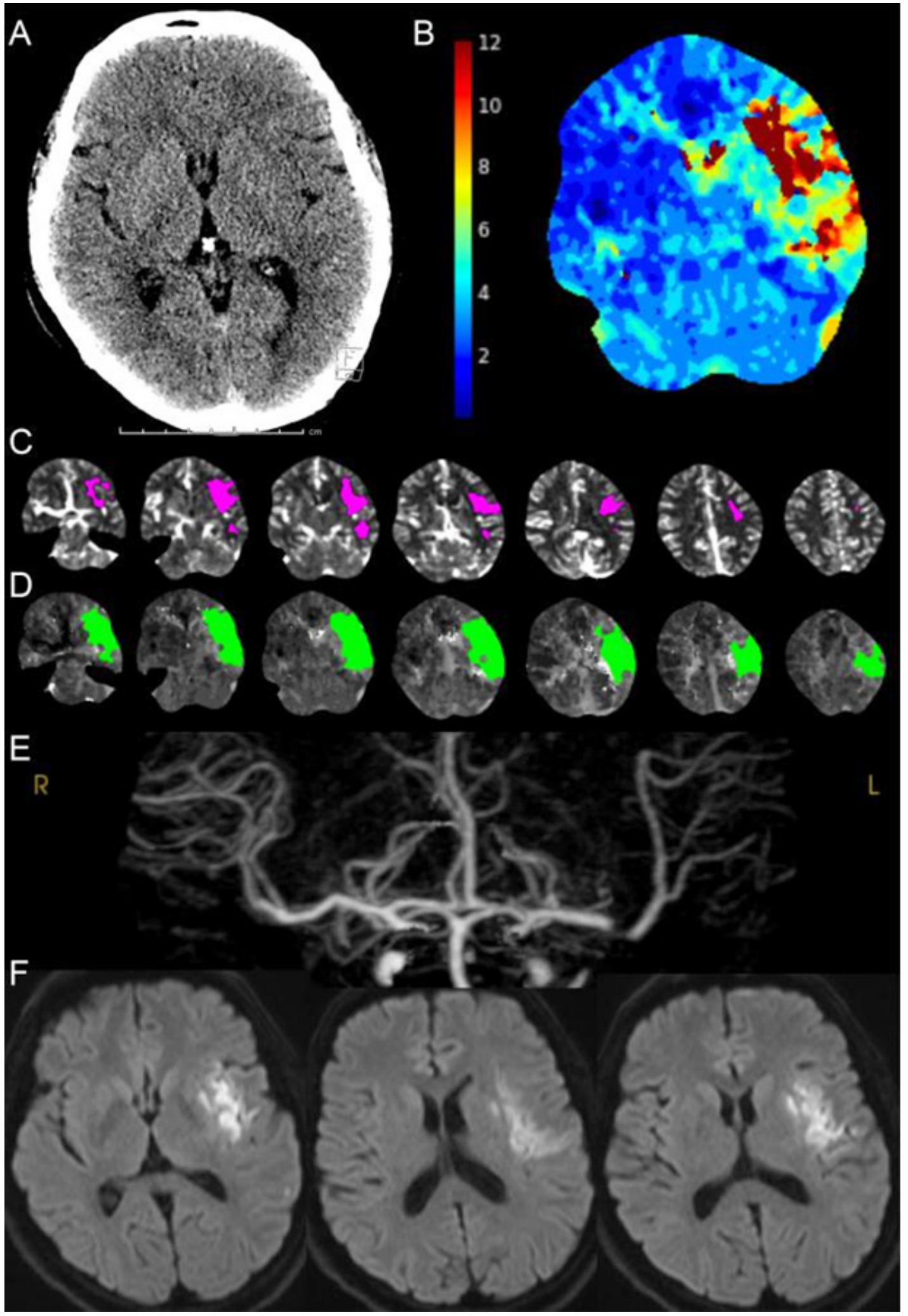

Figure 1. A patient with wake-up onset stroke. Non-contrast CT excluded hemorrhage and indicated subtle loss of grey-white differentiation in the left insular region (A); CT perfusion time to maximum (Tmax) map showing delayed flow via collaterals in the left middle cerebral artery territory confirmed the diagnosis of ischemic stroke (B); Automated segmentation of CT perfusion cerebral blood flow $<30 \%$ of normal brain (36 mL magenta region estimated as irreversibly injured ischemic core) (C) and Tmax $>6$-s delay (124 $\mathrm{mL}$ green region estimated tissue at risk) from RAPID software (D); CT angiography demonstrating distal M1 middle cerebral artery occlusion (E) 24-h diffusion MRI after successful endovascular thrombectomy showing no interval growth in ischemic core (F) 
Thrombolysis (without thrombectomy) in perfusion mismatch-selected patients has proven beneficial 4.5$9 \mathrm{~h}$ after onset, including patients with wake-up stroke who were within $9 \mathrm{~h}$ of the midpoint of sleep ${ }^{[26,27]}$. In practical terms this included wake-up stroke patients up to $16 \mathrm{~h}$ after the time they were last known to be well, similar to the DEFUSE 3 trial time window. The potential for late-window thrombolysis to improve outcomes in combination with thrombectomy is now being explored in randomized trials [Tenecteplase in Stroke Patients Between 4.5 and 24 h (TIMELESS), NCT03785678 and Extending the time window for Tenecteplase by Effective Reperfusion of peNumbrAL tissue in patients with Large Vessel Occlusion (ETERNAL), NCT04454788].

\section{ENDOVASCULAR THROMBECTOMY FOR PATIENTS PRESENTING WITH MILD STROKE DEFICITS OR DISTAL OCCLUSIONS}

The benefit of endovascular thrombectomy in patients presenting with anterior circulation LVO and mild deficits is still unknown, with less than $1 \%$ of patients enrolled in recent thrombectomy trials having a NIHSS $\leq 5^{[28]}$. Despite this cohort having only mild symptoms on first assessment, evidence suggests that without reperfusion therapy, a substantial proportion subsequently deteriorate and are disabled at 90 days (mRS 2-6;29\%) ${ }^{[29,30]}$. Patients with more proximal occlusions, particularly terminal internal carotid artery occlusions, are at the highest risk of neurological deterioration. Deterioration is likely due to leptomeningeal collateral circulation failure over time, in the absence of reperfusion ${ }^{[31]}$. Recent observational data indicate that immediate thrombectomy for the mild stroke patient $(\mathrm{NIHSS} \leq 5)$ is safe and may improve clinical outcomes ${ }^{[28]}$. This is currently being assessed in two randomized controlled trials [Endovascular Therapy for Low NIHSS Ischemic Strokes (ENDOLOW), NCT04167527 and Exploration of the limits of mechanical thrombectomy indications in a single action - MinOr Stroke Therapy Evaluation NIHSS 0-5 (IN EXTREMIS-MOSTE), NCT03796468].

In contrast to the unequivocal evidence for the effectiveness of thrombectomy in patients with proximal occlusion, evidence of benefit beyond the M1 segment is less robust. Relatively few patients with M2 occlusions were included in randomized trials and the definition of M2 segments varied, with many representing early bifurcation of the M1 segment. Anterior cerebral artery and posterior cerebral artery occlusions were not included in trials, other than 3 patients in MR CLEAN. Hypothetically, the benefit of thrombectomy should be reduced given the smaller territory supplied by more distal vessels and the increased efficacy of thrombolysis in reperfusing more distal occlusions. Furthermore, the risk of arterial injury may potentially be increased given the smaller vessel size and increased tortuosity. However, metaanalyses have suggested benefit of thrombectomy in proximal M2 segments in carefully selected patients with significant neurological deficits ${ }^{[15,32]}$. Advances in device technology are likely to improve the safety and efficacy of distal thrombectomy. Further research is needed in this area.

\section{ENDOVASCULAR THROMBECTOMY FOR PATIENTS PRESENTING WITH LARGE ISCHEMIC CORE VOLUMES}

Increasing ischemic core volume (estimated by diffusion restriction on MRI or critically reduced cerebral blood flow on CT perfusion) is associated with lower likelihood of functional independence ${ }^{[33]}$. Despite this association, there is emerging evidence for the benefit of thrombectomy in selected patients with large cores $(>70 \mathrm{~mL})$, particularly within $6 \mathrm{~h}$ of stroke onset ${ }^{[3,34]}$. These data suggest that even with large baseline core volumes, there may be significant volumes of viable but critically hypoperfused tissue that can be salvaged with intervention and translate to clinically meaningful benefit ${ }^{[34]}$. Several randomized controlled trials [Thrombectomy for Emergent Salvage of Large Anterior Circulation Ischemic Stroke (TESLA), NCT03805308; Efficacy and Safety of Thrombectomy in Stroke With Extended Lesion and Extended Time Window (TENSION), NCT03094715; Exploration of the limits of mechanical thrombectomy indications in a single action - Large Stroke Therapy Evaluation - ASPECT 0-5 (IN EXTREMIS - LASTE), NCT03811769 
and Randomized Controlled Trial to Optimize Patient's Selection for Endovascular Treatment in Acute Ischemic Stroke (SELECT-2), NCT03876457] are currently underway to assess this possible benefit. Undoubtedly, rates of functional independence (defined as mRS 0-2) will be substantially lower compared to the imaging-selected randomized controlled thrombectomy trials, such as EXTEND-IA, DAWN and DEFUSE 3. However, mRS 3 outcomes that allow patients to return home with some supports are clinically and economically meaningful compared to death and requirement for fulltime nursing care. Another potential positive outcome may be a reduction in the requirement for hemicraniectomy.

\section{ENDOVASCULAR THROMBECTOMY FOR BASILAR ARTERY OCCLUSIONS}

Basilar artery occlusion is associated with very high levels of morbidity and mortality. Meta-analysis of observational data demonstrated lower rates of death $(\mathrm{HR}=0.49,95 \% \mathrm{CI}$ : 0.44-0.55) and improved modified Rankin scale 4-6 ( $\mathrm{HR}=0.67,95 \% \mathrm{CI}$ : 0.63-0.72) with thrombectomy as compared to best medical management ${ }^{[35]}$. Unfortunately, prospective randomized controlled data are less robust. The BEST randomized trial demonstrated improved outcomes with thrombectomy in an as-treated analysis (mRS 0-2; 39 vs. $19 \%$, OR $=2.81,95 \% \mathrm{CI}: 1.23-6.41)$ but was confounded by high crossover rate from medical management to thrombectomy, resulting in a neutral intention-to-treat analysis ${ }^{[36]}$. The BASICS (Basilar Artery International Cooperation Study) randomized trial has been reported in abstract form and did not demonstrate a significant benefit in favorable outcome with thrombectomy (mRS $\leq 3 ; 44.2$ vs. 37.7\%, RR 1.18, 95\%CI: 0.92-1.50). However, the subgroup with severe clinical deficit (NIHSS $\geq 10$ ) appeared to benefit. Critical details including the rate of successful reperfusion have not yet been released ${ }^{[37]}$.

\section{THROMBOLYSIS FOLLOWED BY THROMBECTOMY VS. DIRECT THROMBECTOMY}

The current standard of care in a patient presenting with a LVO, even in a thrombectomy-capable center, is to give thrombolysis prior to proceeding with thrombectomy. Thrombolysis is more widely available than thrombectomy and should be given at primary stroke centers, if no contraindications exist, prior to transfer to a thrombectomy-capable center. Studies looking at whether thrombolysis can be withheld in patients who present directly to a thrombectomy-capable center are currently underway [Solitaire With the Intention For Thrombectomy Plus Intravenous t-PA Versus DIRECT Solitaire Stent-retriever Thrombectomy in Acute Anterior Circulation Stroke (SWIFT DIRECT), NCT03192332; A Randomized Controlled Trial of DIRECT Endovascular Clot Retrieval Versus Standard Bridging Thrombolysis With Endovascular Clot Retrieval (DIRECT SAFE), NCT03494920 and Multicenter Randomized CLinical trial of Endovascular treatment for Acute ischemic stroke in the Netherlands investigating the added benefit of intravenous alteplase prior to intra-arterial thrombectomy in stroke patients with an intracranial occlusion of the anterior circulation (MR CLEAN-NO IV), ISRCTN80619088]. The first trial published on this topic, Endovascular Thrombectomy with or without Intravenous Alteplase in Acute Stroke [DIRECT-MT], found similar results between direct thrombectomy and combined thrombolysis-thrombectomy arms. Technically, direct thrombectomy narrowly met the specified non-inferiority margin of the lower bound of the $95 \%$ confidence interval for the common odds ratio $>0.80$. However, this margin was overly generous and does not provide reassurance that omitting thrombolysis would be appropriate, even in patients who directly present to a thrombectomy center ${ }^{[38]}$. The rate of successful reperfusion at end of thrombectomy was numerically higher in the alteplase pre-treated group and there were no significant differences in adverse events, including symptomatic intracerebral hemorrhage, between groups ${ }^{[38]}$. Other factors that confound the interpretation of the direct to thrombectomy trials are the use of alteplase rather than the more effective thrombolytic tenecteplase $\mathrm{e}^{[39,40]}$ and potentially distorted acute stroke workflow. Usually, thrombolysis can be given in parallel with thrombectomy decision-making, whereas in these trials, all imaging must be completed and the patients accepted by the interventionist for thrombectomy before they can be randomized and eventually receive thrombolysis. The delay in commencing thrombolysis reduces its opportunity to induce reperfusion prior to thrombectomy. 


\section{TIME TO TREATMENT IS STILL CRUCIAL: IMPROVING DOOR-TO-REPERFUSION TIMES}

The critically time-dependent benefits of reperfusion therapies are well understood, and streamlining workflow to reduce treatment delays remains central to optimizing patient outcomes. Even though individual patients with excellent collateral flow may have slower infarct progression, there is an overall decrease in the proportion of patients eligible for extended-window thrombectomy on the basis of favorable perfusion imaging as time elapses. This corresponds to the time-dependent cerebral blood flow thresholds for infarction and failure of collateral cerebral circulation over time. A meta-analysis looking at time to effective reperfusion demonstrated that for every 4-min delay from emergency department arrival to substantial endovascular reperfusion time, 1 of every 100 treated patients had a worse disability score (higher score by 1 or more on the $\mathrm{mRS})^{[15]}$. Minimizing time from onset of symptoms to reperfusion therapy is therefore crucial in maximizing the number of patients eligible for reperfusion therapy.

The largest delay in reperfusion therapy is in the pre-hospital setting ${ }^{[15]}$. Several advances have been made in both the pre-hospital and emergency department settings. Paramedic stroke recognition and prehospital notification, whereby stroke centers are notified of a potential stroke patient prior to arrival, have been shown to decrease door-to-imaging, door-to-thrombolysis and onset-to-thrombolysis times, while also increasing eligibility for thrombolysis ${ }^{[41-43]}$. In addition, screening tools have been developed to identify suspected LVOs in the pre-hospital setting ${ }^{[44-46]}$. These tools allow patients with suspected LVOs to be transferred directly to thrombectomy-capable centers, bypassing hospitals not capable of providing this service and subsequently improving time to reperfusion. One study investigating an ambulance pre-hospital clinical triage tool demonstrated high sensitivity (85.7\%), specificity $(93.5 \%)$, and positive predictive value $(80 \%)$ for the recognition of thrombectomy-eligible $\operatorname{LVOs}^{[46]}$. Future studies are investigating the application of these tools to accelerate patient delivery to thrombectomy-capable centers by bypassing hospitals not capable of providing this service [Direct Transfer to an Endovascular Center Compared to Transfer to Closest Stroke Center in Acute Stroke with Suspected Large Vessel Occlusion (RACECAT), NCT02795962 and Treatment Strategy In Acute Ischemic large Vessel STROKE: Prioritize Thrombolysis or Endovascular Treatment (TRIAGE), NCT03542188].

There is evidence for the benefit of mobile stroke units (MSUs) in reducing time to reperfusion and subsequent disability. An MSU is an ambulance with on-board CT-scanner and a specialized stroke team capable of assessing and treating patients in the community and directing those eligible to thrombectomycapable centers. A study from Melbourne, Australia demonstrated that the MSU model resulted in an overall time saving from first ambulance dispatch to thrombolysis of $42.5 \mathrm{~min}$ (95\%CI: 36.0-49.0) and a median time saving from first ambulance dispatch to the start of thrombectomy (arterial puncture) of $51 \mathrm{~min}$ in those with LVO (95\%CI: 30.1-71.9 $)^{[47]}$. The estimated disability reduction, based on time saved, was 20.9 disability-adjusted life years for 100 patients in the thrombolysis group and 24.6 disability-adjusted life years in the thrombectomy group ${ }^{[47]}$. Berlin has three MSU and results presented in abstract form indicated improved functional outcomes compared to standard in-hospital care ${ }^{[48]}$.

Workflow efficiencies in the movement of stroke patients in and between hospitals have decreased time to reperfusion therapy in stroke centers around the world. Universal features of efficient systems include emergency department and stroke team prenotification of suspected stroke patients by ambulance, direct transfer of patients from triage to CT table on the ambulance stretcher and the delivery of thrombolysis, if eligible, on the CT table. These interventions have been shown to reduce door-to-thrombolysis to 20$34 \min ^{[49-51]}$. Repeated imaging after transfer is a major cause of delays ${ }^{[52]}$. A time saving of 59 min from door-to-groin access (at a thrombectomy center) is possible if patients diagnosed with LVOs at an external site are transferred directly to the neuro-angiography suite rather than being admitted via the emergency department ${ }^{[53]}$. Another strategy to achieve time savings that is currently under investigation is to transfer patients with suspected LVO (NIHSS score $>10$ on arrival) directly from triage to the neuro-angiography 
suite, bypassing both the emergency department and conventional CT scanner ${ }^{[54,55]}$. A flat panel CT using angiography equipment (or in some cases a separate CT scanner in the angio-suite) can be used to exclude intracerebral hemorrhage, followed by diagnostic angiography and treatment if a LVO is detected. In one observational study, this resulted in significantly shorter median door-to-arterial access times (16 min vs. $70 \mathrm{~min} ; P<0.01$ ) and greater functional independence at 90 days (defined as mRS 0-2; 41 vs. 28\%; $P$ $=0.05)^{[55]}$. However, whether this is an efficient use of limited angiography suite resources remains to be determined.

\section{CONCLUSIONS AND FUTURE DIRECTIONS}

Effective and rapid reperfusion remains the only proven approach to reduce disability in ischemic stroke patients. Thrombectomy has been a major advance, and the indications continue to broaden. Device evolution is likely to continue and the emphasis for technical procedural success is now on rapid "first pass" near-complete reperfusion ${ }^{[56]}$. Mild stroke, distal occlusion and patients with large ischemic core are the current frontiers on which randomized trials are focused. Neuroprotection may yet prove beneficial with a recent trial of nerinetide being neutral overall but suggesting benefit in patients who did not receive thrombolysis ${ }^{[57]}$. Systems of care innovations to accelerate treatment are highly achievable and of critical importance to continue to reduce the disability associated with LVO ischemic stroke.

\section{DECLARATIONS}

\section{Authors' contributions}

Made substantial contributions to conception and design of the commentary and performed data interpretation: Linger M, Campbell BCV

\section{Availability of data and materials}

Not applicable.

\section{Financial support and sponsorship}

None.

\section{Conflicts of interest}

All authors declared that there are no conflicts of interest.

\section{Ethical approval and consent to participate}

Not applicable.

\section{Consent for publication}

Not applicable.

\section{Copyright}

(c) The Author(s) 2020.

\section{REFERENCES}

1. Berkhemer OA, Fransen PS, Beumer D, et al; MR CLEAN Investigators. A randomized trial of intraarterial treatment for acute ischemic stroke. N Engl J Med 2015;372:11-20.

2. Campbell BC, Mitchell PJ, Kleinig TJ, et al; EXTEND-IA Investigators. Endovascular therapy for ischemic stroke with perfusionimaging selection. N Engl J Med 2015;372:1009-18.

3. Goyal M, Demchuk AM, Menon BK, et al; ESCAPE Trial Investigators. Randomized assessment of rapid endovascular treatment of ischemic stroke. N Engl J Med 2015;372:1019-30.

4. Saver JL, Goyal M, Bonafe A, et al; SWIFT PRIME Investigators. Stent-retriever thrombectomy after intravenous t-PA vs. t-PA alone in stroke. N Engl J Med 2015;372:2285-95. 
5. Jovin TG, Chamorro A, Cobo E, et al; REVASCAT Trial Investigators. Thrombectomy within 8 hours after symptom onset in ischemic stroke. N Engl J Med 2015;372:2296-306.

6. Goyal M, Menon BK, van Zwam WH, et al. Endovascular thrombectomy after large-vessel ischaemic stroke: a meta-analysis of individual patient data from five randomised trials. Lancet 2016;387:1723-31.

7. Broderick JP, Palesch YY, Demchuk AM, et al; Interventional Management of Stroke (IMS) III Investigators. Endovascular therapy after intravenous t-PA versus t-PA alone for stroke. N Engl J Med 2013;368:893-903.

8. Ciccone A, Valvassori L, Nichelatti M, et al; SYNTHESIS Expansion Investigators. Endovascular treatment for acute ischemic stroke. $N$ Engl J Med 2013;368:904-13.

9. Kidwell CS, Jahan R, Gornbein J, et al; MR RESCUE Investigators. A trial of imaging selection and endovascular treatment for ischemic stroke. N Engl J Med 2013;368:914-23.

10. Campbell BCV, De Silva DA, Macleod MR, et al. Ischaemic stroke. Nat Rev Dis Primers 2019;5:70.

11. Bracard S, Ducrocq X, Mas JL, et al. Mechanical thrombectomy after intravenous alteplase versus alteplase alone after stroke (THRACE): a randomised controlled trial. Lancet Neurol 2016;15:1138-47.

12. Muir KW, Ford GA, Messow CM, et al; PISTE Investigators. Endovascular therapy for acute ischaemic stroke: the pragmatic ischaemic stroke thrombectomy evaluation (PISTE) randomised, controlled trial. J Neurol Neurosurg Psychiatry 2017;88:38-44.

13. Martins SO, Mont'Alverne F, Rebello LC, et al; RESILIENT Investigators. Thrombectomy for stroke in the public health care system of Brazil. N Engl J Med 2020;382:2316-26.

14. Fransen PS, Berkhemer OA, Lingsma HF, et al; Multicenter Randomized Clinical Trial of Endovascular Treatment of Acute Ischemic Stroke in the Netherlands Investigators. Time to reperfusion and treatment effect for acute ischemic stroke: a randomized clinical trial. JAMA Neurol 2016;73:190-6.

15. Saver JL, Goyal M, van der Lugt A, et al; HERMES Collaborators. Time to treatment with endovascular thrombectomy and outcomes from ischemic stroke: a meta-analysis. JAMA 2016;316:1279-88.

16. Nogueira RG, Jadhav AP, Haussen DC, et al; DAWN Trial Investigators. Thrombectomy 6 to 24 hours after stroke with a mismatch between deficit and infarct. $N$ Engl J Med 2018;378:11-21.

17. Campbell BC, Christensen S, Levi CR, et al. Comparison of computed tomography perfusion and magnetic resonance imaging perfusiondiffusion mismatch in ischemic stroke. Stroke 2012;43:2648-53.

18. Campbell BC, Christensen S, Levi CR, et al. Cerebral blood flow is the optimal CT perfusion parameter for assessing infarct core. Stroke 2011;42:3435-40.

19. Hoving JW, Marquering HA, Majoie CBLM, et al. Volumetric and spatial accuracy of computed tomography perfusion estimated ischemic core volume in patients with acute ischemic stroke. Stroke 2018;49:2368-75.

20. d'Esterre CD, Boesen ME, Ahn SH, et al. Time-dependent computed tomographic perfusion thresholds for patients with acute ischemic stroke. Stroke 2015;46:3390-7.

21. Cereda CW, Christensen S, Campbell BCV, et al. A benchmarking tool to evaluate computer tomography perfusion infarct core predictions against a DWI standard. J Cereb Blood Flow Metab 2016;36:1780-9.

22. Albers GW, Marks MP, Kemp S, et al; DEFUSE 3 Investigators. Thrombectomy for stroke at 6 to 16 hours with selection by perfusion imaging. N Engl J Med 2018;378:708-18.

23. Stroke Foundation (Australia). Clinical guidelines for stroke management. Available from: https://informme.org.au/en/Guidelines/ Clinical-Guidelines-for-Stroke-Management. [Last accessed on 12 Nov 2020]

24. Powers WJ, Rabinstein AA, Ackerson T, et al; American Heart Association Stroke Council. 2018 Guidelines for the early management of patients with acute ischemic stroke: a guideline for healthcare professionals from the american heart association/American stroke association. Stroke 2018;49:e46-110.

25. Desai SM, Haussen DC, Aghaebrahim A, et al. Thrombectomy 24 hours after stroke: beyond DAWN. J Neurointerv Surg 2018;10:1039-42.

26. Ma H, Campbell BCV, Parsons MW, et al; EXTEND Investigators. Thrombolysis guided by perfusion imaging up to 9 hours after onset of stroke. N Engl J Med 2019;380:1795-803.

27. Campbell $\mathrm{BCV}, \mathrm{Ma} \mathrm{H}$, Ringleb PA, et al. Extending thrombolysis to $4 \cdot 5-9 \mathrm{~h}$ and wake-up stroke using perfusion imaging: a systematic review and meta-analysis of individual patient data. Lancet 2019;394:139-47.

28. Nagel S, Bouslama M, Krause LU, et al. Mechanical thrombectomy in patients with milder strokes and large vessel occlusions. Stroke 2018;49:2391-7.

29. Khatri P, Conaway MR, Johnston KC; Acute Stroke Accurate Prediction Study (ASAP) Investigators. Ninety-day outcome rates of a prospective cohort of consecutive patients with mild ischemic stroke. Stroke 2012;43:560-2.

30. Haussen DC, Bouslama M, Grossberg JA, et al. Too good to intervene? Thrombectomy for large vessel occlusion strokes with minimal symptoms: an intention-to-treat analysis. J Neurointerv Surg 2017;9:917-21.

31. Campbell BC, Christensen S, Tress BM, et al; EPITHET Investigators. Failure of collateral blood flow is associated with infarct growth in ischemic stroke. J Cereb Blood Flow Metab 2013;33:1168-72.

32. Menon BK, Hill MD, Davalos A, et al. Efficacy of endovascular thrombectomy in patients with M2 segment middle cerebral artery occlusions: meta-analysis of data from the HERMES Collaboration. J Neurointerv Surg 2019;11:1065-9.

33. Campbell BCV, Majoie CBLM, Albers GW, et al. Penumbral imaging and functional outcome in patients with anterior circulation ischaemic stroke treated with endovascular thrombectomy versus medical therapy: a meta-analysis of individual patient-level data. Lancet Neurol 2019;18:46-55.

34. Rebello LC, Bouslama M, Haussen DC, et al. Endovascular treatment for patients with acute stroke who have a large ischemic core and 
large mismatch imaging profile. JAMA Neurol 2017;74:34-40.

35. Kumar G, Shahripour RB, Alexandrov AV. Recanalization of acute basilar artery occlusion improves outcomes: a meta-analysis. $J$ Neurointerv Surg 2015;7:868-74.

36. Liu X, Dai Q, Ye R, et al. Endovascular treatment versus standard medical treatment for vertebrobasilar artery occlusion (BEST): an open-label, randomised controlled trial. Lancet Neurol 2020;19:115-22.

37. Schonewille W. BASICS - A randomized acute stroke trial of endovascular therapy in acute basilar artery occlusion. European Stroke Organisation Conference 2020 Large Clinical Trials - Webinar. Available from: https://eso-wso-conference.org/eso-wso-may-webinar/. [Last accessed on 12 Nov 2020]

38. Yang P, Zhang Y, Zhang L, et al; DIRECT-MT Investigators. Endovascular thrombectomy with or without intravenous alteplase in acute stroke. N Engl J Med 2020;382:1981-93.

39. Campbell BCV, Mitchell PJ, Churilov L, et al; EXTEND-IA TNK Investigators. Tenecteplase versus alteplase before thrombectomy for ischemic stroke. N Engl J Med 2018;378:1573-82.

40. Campbell BCV, Mitchell PJ, Churilov L, et al; EXTEND-IA TNK Part 2 investigators. Effect of intravenous tenecteplase dose on cerebral reperfusion before thrombectomy in patients with large vessel occlusion ischemic stroke: the EXTEND-IA TNK part 2 randomized clinical trial. JAMA 2020;323:1257-65.

41. Abdullah AR, Smith EE, Biddinger PD, Kalenderian D, Schwamm LH. Advance hospital notification by EMS in acute stroke is associated with shorter door-to-computed tomography time and increased likelihood of administration of tissue-plasminogen activator. Prehosp Emerg Care 2008;12:426-31.

42. McKinney JS, Mylavarapu K, Lane J, Roberts V, Ohman-Strickland P, Merlin MA. Hospital prenotification of stroke patients by emergency medical services improves stroke time targets. J Stroke Cerebrovasc Dis 2013;22:113-8.

43. Lin CB, Peterson ED, Smith EE, et al. Emergency medical service hospital prenotification is associated with improved evaluation and treatment of acute ischemic stroke. Circ Cardiovasc Qual Outcomes 2012;5:514-22.

44. Noorian AR, Sanossian N, Shkirkova K, et al; FAST-MAG Trial Investigators and Coordinators. Los Angeles motor scale to identify large vessel occlusion: prehospital validation and comparison with other screens. Stroke 2018;49:565-72.

45. Pérez de la Ossa N, Carrera D, Gorchs M, et al. Design and validation of a prehospital stroke scale to predict large arterial occlusion: the rapid arterial occlusion evaluation scale. Stroke 2014;45:87-91.

46. Zhao H, Pesavento L, Coote S, et al. Ambulance clinical triage for acute stroke treatment: paramedic triage algorithm for large vessel occlusion. Stroke 2018;49:945-51.

47. Zhao H, Coote S, Easton D, et al. Melbourne mobile stroke unit and reperfusion therapy: greater clinical impact of thrombectomy than thrombolysis. Stroke 2020;51:922-30.

48. Audebert HJ. The effects of mobile stroke units on functional outcome after acute cerebral ischemia (B_PROUD). 2020. Available from: https:/professional.heart.org/idc/groups/ahamah-public/@wcm/@sop/@scon/documents/downloadable/ucm_505623.pdf. [Last accessed on 12 Nov 2020]

49. Meretoja A, Weir L, Ugalde M, et al. Helsinki model cut stroke thrombolysis delays to 25 minutes in Melbourne in only 4 months. Neurology 2013;81:1071-6.

50. Meretoja A, Strbian D, Mustanoja S, Tatlisumak T, Lindsberg PJ, Kaste M. Reducing in-hospital delay to 20 minutes in stroke thrombolysis. Neurology 2012;79:306-13.

51. Wu TY, Coleman E, Wright SL, et al. Helsinki stroke model is transferrable with "real-world" resources and reduced stroke thrombolysis delay to $34 \mathrm{~min}$ in christchurch. Front Neurol 2018;9:290.

52. Campbell BCV. Optimal imaging at the primary stroke center. Stroke 2020;51:1932-40.

53. Jadhav AP, Kenmuir CL, Aghaebrahim A, et al. Interfacility transfer directly to the neuroangiography suite in acute ischemic stroke patients undergoing thrombectomy. Stroke 2017;48:1884-9.

54. Ribo M, Boned S, Rubiera M, et al. Direct transfer to angiosuite to reduce door-to-puncture time in thrombectomy for acute stroke. $J$ Neurointerv Surg 2018;10:221-4.

55. Mendez B, Requena M, Aires A, et al. Direct transfer to angio-suite to reduce workflow times and increase favorable clinical outcome. Stroke 2018;49:2723-7.

56. Nikoubashman O, Dekeyzer S, Riabikin A, et al. True first-pass effect. Stroke 2019;50:2140-6.

57. Hill MD, Goyal M, Menon BK, et al. Efficacy and safety of nerinetide for the treatment of acute ischaemic stroke (ESCAPE-NA1): a multicentre, double-blind, randomised controlled trial. Lancet 2020;395:878-87. 